\title{
A Comparison of Quantum Oracles
}

\author{
Elham Kashefi*†, Adrian Kent ${ }^{\mathcal{S}}$, Vlatko Vedral* and Konrad Banaszek ${ }^{* \dagger}$ \\ * Optics Section, The Blackett Laboratory, Imperial College, London SW7 2BZ, England \\ ${ }^{\dagger}$ Centre for Quantum Computation, Clarendon Laboratory, University of Oxford, Parks Road, Oxford OX1 3PU, England \\ ${ }^{\mathcal{S}}$ Hewlett-Packard Laboratories, Filton Road, Stoke Gifford, Bristol BS34 8QZ, England
}

(October 31, 2018)

\begin{abstract}
A standard quantum oracle $S_{f}$ for a general function $f: Z_{N} \rightarrow Z_{N}$ is defined to act on two input states and return two outputs, with inputs $|i\rangle$ and $|j\rangle\left(i, j \in Z_{N}\right)$ returning outputs $|i\rangle$ and $|j \oplus f(i)\rangle$. However, if $f$ is known to be a one-to-one function, a simpler oracle, $M_{f}$, which returns $|f(i)\rangle$ given $|i\rangle$, can also be defined. We consider the relative strengths of these oracles. We define a simple promise problem which minimal quantum oracles can solve exponentially faster than classical oracles, via an algorithm which cannot be naively adapted to standard quantum oracles. We show that $S_{f}$ can be constructed by invoking $M_{f}$ and $\left(M_{f}\right)^{-1}$ once each, while $\Theta(\sqrt{N})$ invocations of $S_{f}$ and/or $\left(S_{f}\right)^{-1}$ are required to construct $M_{f}$.
\end{abstract}

Recent years have witnessed an explosion of interest in quantum computation, as it becomes clearer that quantum algorithms are more efficient than any known classical algorithm for a variety of tasks. 11 4. One important way of comparing the efficiencies is by analysing query complexity, which measures the number of invocations of an "oracle" — which may be a standard circuit implementing a useful sub-routine, a physical device, or a purely theoretical construct - needed to complete a task. A number of general results show the limitations and advantages of quantum computers using the query complexity models [5].

In this paper we compare the query complexity analysis of quantum algorithms given two different ways of representing a permutation in terms of a black box quantum oracle. We begin with a short discussion of graph isomorphism problems, which motivates the rest of the paper.

Suppose we are given two graphs, $G_{1}=\left(V_{1}, E_{1}\right)$ and $G_{2}=\left(V_{2}, E_{2}\right)$, represented as sets of vertices and edges in some standard notation. The graph isomorphism (GI) problem is to determine whether $G_{1}$ and $G_{2}$ are isomorphic: that is, whether there is a bijection $f: V_{1} \rightarrow V_{2}$ such that $(f(u), f(v)) \in E_{2}$ if and only if $(u, v) \in E_{1}$. (We assume $\left|V_{1}\right|=\left|V_{2}\right|$, else the problem is trivial.) GI is a problem which is NP but not known to be NP-complete for classical computers, and for which no polynomial time quantum algorithm is currently known.

We are interested in a restricted version (NAGI) of GI, in which it is given that $G_{1}$ and $G_{2}$ are non-automorphic: i.e., they have no non-trivial automorphisms. So far as we are aware, no polynomial time classical or quantum algorithms are known for NAGI either. The following observations suggest a possible line of attack in the quantum case.

First, for any non-automorphic graph $G=(V, E)$, we can define a unitary map $M_{G}$ that takes permutations $\rho$ of $V$ as inputs and outputs the permuted graph $\rho(G)=(\rho(V), \rho(E))$, with some standard ordering (e.g. alphabetical) of the vertices and edges, in some standard computational basis representations. That is, writing
$|V|=N$, for any $\rho \in S_{N}, M_{G}$ maps $|\rho\rangle$ to $|\rho(G)\rangle$. Consider a pair $\left(G_{1}, G_{2}\right)$ of non-automorphic graphs. Given circuits implementing $M_{G_{1}}, M_{G_{2}}$, we could input copies of the state $\frac{1}{\sqrt{N !}} \sum_{\rho \in S_{N}}|\rho\rangle$ to each circuit, and compare the outputs $\left|\psi_{i}\right\rangle=\frac{1}{\sqrt{N !}} \sum_{\rho \in S_{N}}\left|\rho\left(G_{i}\right)\right\rangle$. Now, if the graphs are isomorphic, these outputs are equal; if not, they are orthogonal. These two cases can be distinguished with arbitrarily high confidence in polynomial time (see below), so this would solve the problem.

Unfortunately, our algorithm for NAGI requires constructing circuits for the $M_{G_{i}}$, which could be at least as hard as solving the original problem. On the other hand, it is easy to devise a circuit, $S_{G}$, which takes two inputs, $|\rho\rangle$ and a blank set of states $|0\rangle$, and outputs $|\rho\rangle$ and $|\rho(G)\rangle$. Since $S_{G}$ and $M_{G}$ implement apparently similar tasks, one might hope to find a way of constructing $M_{G}$ from a network involving a small number of copies of $S_{G}$. Such a construction would solve NAGI. Alternatively, one might hope to prove such a construction is impossible, and so definitively close off this particular line of attack.

Thus motivated, we translate this into an abstract problem in query complexity.

Consider the following oracles, defined for a general function $f:\{0,1\}^{m} \rightarrow\{0,1\}^{n}$ :

- the standard oracle, $S_{f}:|x\rangle|b\rangle \rightarrow|x\rangle|b \oplus f(x)\rangle$.

- the Fourier phase oracle, $P_{f}:|x\rangle|b\rangle \rightarrow$ $e^{2 \pi i f(x) b / 2^{n}}|x\rangle|b\rangle$.

Here $x$ and $b$ are strings of $m$ and $n$ bits respectively, represented as numbers modulo $M=2^{m}$ and $N=2^{n},|x\rangle$ and $|b\rangle$ are the corresponding computational basis states, and $\oplus$ is addition modulo $2^{n}$.

Note that the oracles $P_{f}$ and $S_{f}$ are equivalent, in the sense that each can be constructed by an $f$-independent quantum circuit containing just one copy of the other, and also equivalent to their inverses. To see this, define the quantum Fourier transform operation $F$ and the 
parity reflection $R=F^{2}$ by

$$
F:|j\rangle \rightarrow \frac{1}{\sqrt{N}} \sum_{k=0}^{N-1} \exp (2 \pi i j k / N)|k\rangle, \quad R:|j\rangle \rightarrow|-j\rangle .
$$

Then we have

$$
\begin{aligned}
& (I \otimes F) \circ S_{f} \circ\left(I \otimes F^{-1}\right)=P_{f}, \\
& \left(I \otimes F^{-1}\right) \circ P_{f} \circ(I \otimes F)=S_{f}, \\
& (I \otimes R) \circ S_{f} \circ(I \otimes R)=\left(S_{f}\right)^{-1}, \\
& (I \otimes R) \circ P_{f} \circ(I \otimes R)=\left(P_{f}\right)^{-1} .
\end{aligned}
$$

For the rest of the paper we take $m=n$ and suppose we know $f$ is a permutation on the set $\{0,1\}^{n}$. There is then a simpler invertible quantum map associated to $f$ :

- the minimal oracle: $M_{f}:|x\rangle \rightarrow|f(x)\rangle$.

We can model NAGI, and illustrate the different behaviour of standard and minimal oracles, by a promise problem. Suppose we are given two permutations, $\alpha$ and $\beta$, of $Z_{N}$, and a subset $S$ of $Z_{N}$, and are promised that the images $\alpha(S)$ and $\beta(S)$ are either identical or disjoint. The problem is to determine which. (This problem has been considered in a different context by Buhrman et al [8].)

We represent elements $x \in Z_{N}$ by computational basis states of $n$ qubits in the standard way, and write $|S\rangle=\sum_{x \in S}|x\rangle$.

Figure 1 gives a quantum network with minimal oracles that identifies disjoint images with probability at least $1 / 2$.



FIG. 1. A quantum circuit for the permutation promise problem. $O_{\alpha}$ and $O_{\beta}$ are minimal oracles for computing the permutations $\alpha$ and $\beta$ respectively, $|S\rangle$ is the superposition of all the basis states, $H$ is the Hadamard transformation, and all the other gates are conditional swap gates, where circles signify control bits.

Let $A=\{\alpha(x) \mid x \in S\}$ and $B=\{\beta(x) \mid x \in S\}$. One query to the oracles $M_{\alpha}$ and $M_{\beta}$ creates the (unnormalised) states $|A\rangle$ and $|B\rangle$ respectively. The state before applying the controlled gates is:

$$
|A\rangle|B\rangle \otimes(|0\rangle-|1\rangle)
$$

After controlled swap gates, the state becomes:

$$
|A\rangle|B\rangle|0\rangle-|B\rangle|A\rangle|1\rangle .
$$

The final Hadamard gate on the ancilla qubit gives:

$$
(|A\rangle|B\rangle-|B\rangle|A\rangle)|0\rangle+(|A\rangle|B\rangle+|B\rangle|A\rangle)|1\rangle
$$

A $|0\rangle$ outcome shows unambiguously that the images are disjoint. A $|1\rangle$ outcome is generated with probability 1 if the images are identical, and with probability $1 / 2$ if the images are disjoint. Repeating the computation $K$ times allows one to exponentially improve the confidence of the result. If after $K$ trials we get $|0\rangle$ at least once, we know for certain that $\alpha(S) \neq \beta(S)$. When all the $K$ outcomes were $|1\rangle$, the conclusion that $\alpha(S)=\beta(S)$ has the conditional probability $p_{K}=\frac{1}{2^{K}}$ of having been erroneously generated by disjoint input images. Note that $p_{K}$ is independent of the problem size and decreases exponentially with the number of repetitions.

Clearly, a naive adaptation of the algorithm to standard oracles does not work. Replacing $M_{\alpha}$ and $M_{\beta}$ by $S_{\alpha}$ and $S_{\beta}$, and replacing the inputs by $|S\rangle \otimes|0\rangle$, results in output states which are orthogonal if the images are disjoint, but also in general very nearly orthogonal if the images are identical. Applying a symmetric projection as above thus almost always fails to distinguish the cases. To the best of our knowledge a non-trivial lower bound for this problem using the $S_{f}$ is not known (however, see [9]).

This example suggests that minimal oracles may be rather more powerful than standard oracles. To establish a more precise version of this hypothesis, we examine how good each oracle is at simulating the other. One way round turns out to be simple. We can construct $S_{f}$ from $M_{f}$ and $\left(M_{f}\right)^{-1}=M_{f-1}$ as follows:

$$
S_{f}=\left(M_{f^{-1}} \otimes I\right) \circ A \circ\left(M_{f} \otimes I\right)
$$

where $\circ$ represents the composition of operations (or the concatenation of networks) and the modulo $N$ adder $A$ is defined by $A:|a\rangle \otimes|b\rangle \rightarrow|a\rangle \otimes|a \oplus b\rangle$.

Suppose that we are given $M_{f}$ in the form of a specified complicated quantum circuit. We may be completely unable to simplify the circuit or deduce a simpler form of $f$ from it. However, by reversing the circuit gate by gate, we can construct a circuit for $\left(M_{f}\right)^{-1}$. Hence, by the above construction, we can produce a circuit for $S_{f}$, using one copy and one reversed copy of the circuit for $M_{f}$.

This way of looking at oracles can be formalised into the circuit model, in which the query complexity of an algorithm involving an oracle $O_{f}$ associated to a function $f$ is the number of copies of $O_{f}$ and/or $O_{f}^{-1}$ required to implement the algorithm in a circuit that, apart from the oracles, is independent of $f$. In the circuit model, a standard oracle can easily be simulated given a minimal oracle. Ignoring constant factors, we say that the minimal oracle is at least as strong as the standard oracle. 
It should be stressed that, while the circuit model has a natural justification, there are other interesting oracle models, to which our arguments will not apply. For example, if we think of the oracle $M_{f}$ as a black box supplied by a third party, then we should not assume that $\left(M_{f}\right)^{-1}$ can easily be constructed from $M_{f}$, as we know no way of efficiently reversing the operation of an unknown physical evolution.

Remaining within the circuit model, we now show that $M_{f}$ and $S_{f}$ are not (even up to constant factors) equivalent. In fact, simulating $M_{f}$ requires exponentially many uses of $S_{f}$.

First, consider the standard oracle $S_{f^{-1}}$ which maps a basis state $|y\rangle|b\rangle$ to $|y\rangle\left|b \oplus f^{-1}(y)\right\rangle$. Since $S_{f^{-1}}:|y\rangle|0\rangle \rightarrow$ $|y\rangle\left|f^{-1}(y)\right\rangle$, simulating it allows us to solve the search problem of identifying $\left|f^{-1}(y)\right\rangle$ from a database of $N$ elements. It is known that, using Grover's search algorithm, one can simulate $S_{f^{-1}}$ with $O(\sqrt{N})$ invocations of $S_{f}[10,11]$. In the following we explain one possible way of doing that.

Prepare the state $|y\rangle|0\rangle|0\rangle|0\rangle$, where the first three registers consist of $n$ qubits and the last register is a single qubit. Apply Hadamard transformations on the second register to get $\left|\phi_{1}\right\rangle=|y\rangle \sum_{x \in Z_{N}}|x\rangle|0\rangle|0\rangle$. Invoking $S_{f}$ on the second and third registers now gives

$$
|y\rangle\left(\sum_{x \in Z_{N}}|x\rangle|f(x)\rangle\right)|0\rangle
$$

Using CNOT gates, compare the first and third registers and put the result in the fourth, obtaining

$$
\left(|y\rangle \sum_{x \in Z_{N}, x \neq f^{-1}(y)}|x\rangle|f(x)\rangle|0\rangle\right)+\left(|y\rangle\left|f^{-1}(y)\right\rangle|y\rangle|1\rangle\right) .
$$

Now apply $\left(S_{f}\right)^{-1}$ on the second and third registers, obtaining

$$
\left(|y\rangle \sum_{x \in Z_{N}, x \neq f^{-1}(y)}|x\rangle|0\rangle|0\rangle\right)+\left(|y\rangle\left|f^{-1}(y)\right\rangle|0\rangle|1\rangle\right) .
$$

Taken together, these operations leave the first and third registers unchanged, while their action on the second and fourth defines an oracle for the search problem. Applying Grover's algorithm [3] to this oracle, we obtain the state $|y\rangle\left|f^{-1}(y)\right\rangle$ after $O(\sqrt{N})$ invocations.

Lemma 1 To simulate the inverse oracle $S_{f-1}$ with a quantum network using oracles $S_{f}$ and $\left(S_{f}\right)^{-1}$, a total number of $\Theta(\sqrt{N})$ invocations of $S_{f}$ are necessary.

Proof The upper bound of $O(\sqrt{N})$ is implied by the Grover-based algorithm just discussed. Ambainis 12 has shown that $\Omega(\sqrt{N})$ invocations of the standard oracle $S_{f}$ are required to invert a general permutation $f$. QED.

Given $S_{f}$ and $S_{f-1}$, Bennett has shown how to simulate $M_{f}$ within classical reversible computation 13]. Using a quantum version of this construction, we can establish our main result:
Lemma 2 To simulate the minimal oracle $M_{f}$ with a quantum network using oracles $S_{f}$ and $\left(S_{f}\right)^{-1}$, a total number of $\Theta(\sqrt{N})$ invocations of $S_{f}$ are necessary.

Proof Given $S_{f}$ and $S_{f-1}$, we can simulate $M_{f}$ as follows:

$$
M_{f} \otimes I=\left(S_{f^{-1}}\right)^{-1} \circ X \circ S_{f}
$$

where the swap gate $X$ is defined by $X:|a\rangle \otimes|b\rangle \rightarrow$ $|b\rangle \otimes|a\rangle$. From Lemma $1, S_{f^{-1}}$ needs $\Theta(\sqrt{N})$ invocations of $S_{f}$ and $\left(S_{f}\right)^{-1}$. Therefore we get the upper bound of $O(\sqrt{N})$ for simulation of $M_{f}$.

However this is the optimal simulation. For suppose there is a network which simulates $M_{f}$ with less than $\Omega(\sqrt{N})$ queries. The reversed network simulates $M_{f-1}$. From these two, by our earlier results, we can construct a network that simulates $S_{f^{-1}}$ with fewer than $\Omega(\sqrt{N})$ queries, which contradicts Lemma 1. QED.

It is worth remarking that we could equally well have carried through our discussion using variants of $S_{f}$ and $P_{f}$, such as the bitwise acting versions:

- the bit string standard oracle, $S_{f}^{\text {bit }}:|\mathbf{x}\rangle|\mathbf{b}\rangle \rightarrow$ $|\mathbf{x}\rangle|\mathbf{b} \oplus \mathbf{f}(\mathbf{x})\rangle$.

- the bit string phase oracle, $P_{f}^{\text {bit }}:|\mathbf{x}\rangle|\mathbf{b}\rangle \rightarrow$ $e^{2 \pi i \mathbf{f}(\mathbf{x}) \cdot \mathbf{b} / 2}|\mathbf{x}\rangle|\mathbf{b}\rangle$.

Here $\mathbf{b} \oplus \mathbf{x}$ denotes the bitwise sum mod 2 of the strings $\mathbf{b}$ and $\mathbf{x}$, and $\mathbf{b} \cdot \mathbf{x}$ their inner product $\bmod 2$. Again, $S_{f}^{\text {bit }}$ and $P_{f}^{\text {bit }}$ are equivalent: writing

$$
\mathcal{F}=H \otimes H \otimes \cdots \otimes H
$$

for the tensor product of $n$ Hadamard operators acting on register qubits, we have

$$
\begin{aligned}
& (I \otimes \mathcal{F}) \circ S_{f}^{\mathrm{bit}} \circ\left(I \otimes \mathcal{F}^{-1}\right)=P_{f}^{\mathrm{bit}}, \\
& \left(I \otimes \mathcal{F}^{-1}\right) \circ P_{f}^{\mathrm{bit}} \circ(I \otimes \mathcal{F})=S_{f}^{\mathrm{bit}} .
\end{aligned}
$$

Note also that $S_{f}^{\text {bit }}=\left(S_{f}^{\text {bit }}\right)^{-1}, P_{f}^{\text {bit }}=\left(P_{f}^{\text {bit }}\right)^{-1}$. Our results still apply: $S_{f}^{\text {bit }}$ has essentially the same relation to $M_{f}$ that $S_{f}$ does.

In summary, constructing a minimal oracle requires exponentially many invocations of a standard oracle. We can thus indeed definitively exclude the possibility of efficiently solving NAGI by simulating $M_{f}$ using $S_{f}$, which motivated our discussion. We have not, however, been able to exclude the possibility of directly constructing a polynomial size network defining an $M_{f}$ oracle for any given $1-1$ function $f$, which would lead to a polynomial time solution of NAGI.

Acknowledgments. We thank Charles Bennett for helpful discussions and for drawing our attention to Refs. [13, and Richard Jozsa for helpful comments. E. K. thanks 
Mike Mosca for useful discussions and Waterloo University for hospitality. This work was supported by EPSRC and by the European projects EQUIP, QAIP and QUIPROCONE.

[1] D. Deutsch, Proc. Royal Society of London A, 400, 97 (1985).

[2] P.W. Shor, SIAM J. Comp., 26, 1484 (1997).

[3] L.K. Grover, Proc. 28th ACM Symp. Theor. Comp., 212 (1996).

[4] C. Bennett, E. Bernstein, G. Brassard, and U. Vazirani, SIAM J. Comp., 26, 1510 (1997).

[5] R. Beals, H. Buhrman, R. Cleve, M. Mosca, and R. de Wolf, Proc. 39th Symp. Found. Comp. Sci., 352
(1998).

[6] W. van Dam, Proc. 39th Symp. Found. Comp. Sci., 362 (1998).

[7] R. Cleve, in C. Macchiavello, G.M. Palma, and A. Zeilinger, editors, Collected Papers on Quantum Computation and Quantum Information Theory, (World Scientific, 1999).

[8] H. Buhrman, R. Cleve, J. Watrous, and R. de Wolf, quant-ph/0102001, 2001.

[9] After this work was circulated, a non-trivial lower bound was given by S. Aaronson quant-ph/0111102

[10] G. Brassard, P. Høyer, and A. Tapp, 3rd Latin American Theor. Info. Symp., 1380, 163 (1998).

[11] G. Brassard, P. Høyer, M. Mosca, and A. Tapp, to appear in Quantum Computation \& Quantum Information Science, AMS Contemporary Math Series, quantph/0005055, 2001.

[12] A. Ambainis, Proc. 32th ACM Symp. Theor. Comp., 636 (2000).

[13] C. Bennett, IBM J. Res. Dev., 17, 525 (1973). 